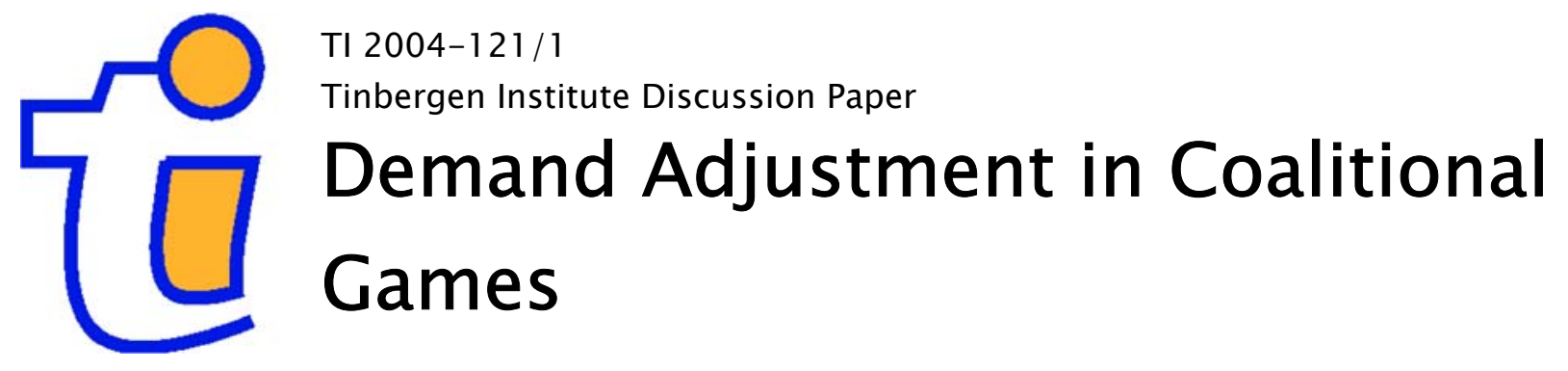
G. van der Laan
V. Pruzhansky

Department of Econometrics, Faculty of Economics and Business Administration, Vrije Universiteit Amsterdam, and Tinbergen Institute. 
Tinbergen Institute

The Tinbergen Institute is the institute for economic research of the Erasmus Universiteit Rotterdam, Universiteit van Amsterdam, and Vrije Universiteit Amsterdam.

Tinbergen Institute Amsterdam

Roetersstraat 31

1018 WB Amsterdam

The Netherlands

Tel.: $\quad+31(0) 205513500$

Fax: $\quad+31(0) 205513555$

Tinbergen Institute Rotterdam

Burg. Oudlaan 50

3062 PA Amsterdam

The Netherlands

Tel.: $\quad+31(0) 104088900$

Fax: $\quad+31(0) 104089031$

Please send questions and/or remarks of nonscientific nature to driessen@tinbergen.nl.

Most TI discussion papers can be downloaded at http://www.tinbergen.nl. 


\title{
DEMAND ADJUSTMENT IN COALITIONAL GAMES
}

\author{
G. VAN DER LAAN AND V. PRUZHANSKY
}

\begin{abstract}
The paper associates a strategic $n$-person game with a given transferable utility game and studies its Nash equilibria. Strict equilibria in this model characterize those divisions of social surplus that can become conventions in the sense of Young [16]. It is shown that even in relatively simple games various inefficiencies can arise.
\end{abstract}

\section{INTRODUCTION}

Imagine a set $N=\{1, \ldots, n\}$ of $n$ players that simultaneously and non-cooperatively submit their claims to divide some worth. Specifically, any group (coalition) $S \subseteq N$ of players can share the worth of $S$, which is given by $v(S) \in \mathbb{R}$, and no player cares which particular coalition satisfies his demand. The notion of worth is typical for cooperative game theory, whereas the notion of simultaneity and independence in submitting claims comes from non-cooperative theory. Such situations represent an interesting mix of both cooperative and non-cooperative aspects of bargaining.

A word of caution is needed. The bargaining process just described is different from the traditional view held by cooperative or non-cooperative game theory. While conventional cooperative bargaining concepts are defined either on the space of aspirations (aspiration bargaining set or partnered aspirations, eg. Bennett [7]) or imputations (eg. bargaining set of Aumann and Maschler [2], kernel, eg. Davis and Maschler [11], etc.), we will show that there exist demand vectors that are stable (in the sense to be defined precisely below), but are neither aspirations, nor imputations. Non-cooperative theory usually models the process of bargaining as an extensive game (eg. Rubinstein [15], Krishna and Serrano [12]), in which players' preferences depend on the time an agreement is reached. In contrast, we will analyze this process

Date: October 8, 2004.

Key words and phrases. Aspirations, bargaining set, core, von NeumannMorgenstern vector, stable set.

We are thankful to René van den Brink, Harold Houba, Jurjen Kamphorst and Maria Montero for their comments. 
as a strategic game. Finally, both cooperative and non-cooperative approaches presuppose that players make proposals to each other regarding the distribution of gains, that is some sort of communication is possible among the agents. We take a more primitive position here. In our model agents cannot communicate directly with each other. One reason for this might be that there are too many agents ${ }^{1}$. Hence they just submit their demands to some central authority, who then determines which coalitions will be formed according to some reasonable criterion (again, to be defined precisely below). It will be shown that the set of Nash equilibria in this game can vastly differ from the set of competitive equilibria; moreover, various inefficiencies can arise.

One of the central questions of cooperative game theory is what coalitions are most reasonable to form. In light of the present paper, it appears that this question is only secondary to a more primitive one. Namely, which claims for the division of the surplus are most reasonable. The following analogy with non-cooperative game theory can be helpful in understanding this. In non-cooperative games the primary focus is on the strategies that players select. Only after reasonable strategies have been singled out, we look for the outcomes that are consistent with the selected strategies. Likewise, in cooperative games we suggests that one first focuses on demands that players find stable (according to some reasonable criterion), and only after that wonders what coalitions can possibly form. That is, demands are the main, while coalition formation is a side issue of the process.

The paper is structured as follows. Section 2 introduces a basic model of demand adjustment and Section 3 discusses related literature. Section 4 presents our key results and Section 5 deals with the case of three-player games. Section 6 considers some further examples, such as market games and apex games.

\section{The Model}

Denote by $N=\{1, \ldots, n\}$ the set of players and by $2^{N}$ the power set of $N$, excluding the empty coalition $\varnothing$. We study transferable utility games (TU-games), in which the possibilities for cooperation by any subset of players $S \subseteq N$ are described by means of a characteristic function $v: 2^{N} \cup \varnothing \rightarrow \mathbb{R}$. The TU-game with player set $N$ and characteristic function $v$ is denoted by $(N, v)$.

We assume that for any $S \in 2^{N}, v(S) \geq 0$ with strict inequality for at least one $S$. Moreover, $v$ is commonly known to all $n$ players. One interpretation of $v(S)$ is that this is the largest monetary gain that

\footnotetext{
${ }^{1}$ See Section 6 for other interpretations.
} 
coalition $S$ can achieve without the cooperation of $N \backslash S$. By normalization, $v(\{i\})=v(\varnothing)=0$ for all $i \in N$. There is no loss of generality in doing so, since we are interested only in pure gains from cooperation. Two players $i$ and $j$ are said to be symmetric if for any $S \in 2^{N}$, such that $i, j \notin S$, we have

$$
v(S \cup\{i\})=v(S \cup\{j\}) .
$$

The game $(N, v)$ is convex if for any two coalitions $S, T \in 2^{N}$ it holds that

$$
v(S)+v(T) \leq v(S \cup T)+v(S \cap T) .
$$

If the above holds for any two disjoint coalitions $S$ and $T$, then the game $(N, v)$ is said to be superadditive. Clearly, any convex game is also superadditive.

A payoff vector of an $n$-person game $(N, v)$ is an $n$-dimensional vector $x \in \mathbb{R}_{+}^{n}$ yielding a payoff $x_{i}$ to each player $i \in N$. For any $S \subseteq N$, let $x(S)=\sum_{i \in S} x_{i}$. A vector $x \in \mathbb{R}^{n}$ is called an imputation iff $\bar{x}(N)=$ $v(N)$ and $x_{i} \geq v(\{i\})=0$ for all $i \in N$. The set of imputations of the game $(N, v)$ will be denoted by $I(N, v)$. A vector $x \in \mathbb{R}^{n}$ is said to be an aspiration if and only if it satisfies the following two conditions:

i) $x(S) \geq v(S)$ for all $S \subseteq N$ (maximality).

ii) for each $i \in N$ there exists a coalition $S \ni i$, such that $x(S) \leq$ $v(S)$ (feasibility).

We will denote the set of aspirations by $A(N, v)$. Observe that for any $x \in A(N, v)$ the conditions (i) and (ii) imply that for each $i \in N$ there exists a coalition $S$ containing $i$ such that $x(S)=v(S)$. Typically $A(N, v)$ is very large. However, not all aspirations are reasonable. With the purpose of further narrowing down this set many 'refinements' of $A(N, v)$ have been proposed in the literature, eg. partnered aspirations (Bennett [3]) or bargaining aspirations (Bennett and Zame [7]). For precise definitions we refer to the original papers.

A well-known solution for transferable utility games is the core. The core is the subset of imputations, defined by

$$
C(N, v)=\left\{x \in \mathbb{R}^{n} \mid x(N)=v(N) \text { and } x(S) \geq v(S) \text { for all } S \in 2^{N}\right\} .
$$

In the sequel we will also use the notion of strict core, defined by

$$
\begin{aligned}
\widetilde{C}(N, v) & =\left\{x \in C(N, v) \mid x_{i}>v(\{i\}) \text { for all } i \in N\right\} \\
& =\left\{x \in C(N, v) \mid x_{i}>0 \text { for all } i \in N\right\},
\end{aligned}
$$

where the second equality follows from the normalization. 
In the following we will introduce a non-cooperative normal form game $\left\langle N,\left\{X_{i}\right\}_{i \in N},\left\{u_{i}\right\}_{i \in N}\right\rangle$ induced by the cooperative game $(N, v)$, where $X_{i}$ is the pure strategy space and $u_{i}$ the (expected) utility function of player $i \in N$. A strategy of player $i$ is a particular monetary claim $x_{i} \in \mathbb{R}_{+}$. The strategy space of $i$ is a convex and compact subset $X_{i}=\left[0, \bar{x}_{i}\right]$ of $\mathbb{R}_{+}$, where $\bar{x}_{i}>\max \{v(S), S \subseteq N\}$. Correspondingly, the strategy space of the game is

$$
X=\times_{i \in N} X_{i} .
$$

Within the non-cooperative framework any $n$-dimensional vector $x \in X$ is, thus, a vector of demands ${ }^{2}$, in which the $i$-th coordinate stands for the demand of player $i .{ }^{3}$ Given a vector of demands $x \in X$, we say that coalition $S$ is feasible if and only if $x(S) \leq v(S)$. Let $\pi=\left\{S^{1}, \ldots, S^{m}\right\}$ be a partition ${ }^{4}$ of $N$, that is the sets $S^{1}, \ldots, S^{m}$ are non-empty, pairwise disjoint and satisfy $\bigcup_{k=1}^{m} S^{k}=N$. Given a demand vector $x$, we say that a partition $\pi(x)=\left\{S^{1}, \ldots, S^{m}\right\}$ is compatible with $x$ if for each $k=1, \ldots, m$ it holds that

$$
\text { either }\left|S^{k}\right|=1 \text { or both }\left|S^{k}\right| \geq 2 \text { and } x\left(S^{k}\right) \leq v\left(S^{k}\right) \text {. }
$$

So, if the partition $\pi(x)$ is compatible with $x$, any coalition $S \in \pi(x)$ containing at least two players must be feasible given $x$. A compatible partition $\pi(x)=\left\{S^{1}, \ldots, S^{m}\right\}$ is called minimal if and only if

$$
x\left(\bigcup_{k \in K} S^{k}\right)>v\left(\bigcup_{k \in K} S^{k}\right) \text { for all } K \subseteq\{1, \ldots, m\} \text { with }|K| \geq 2 .
$$

Thus, for given $x$, a compatible partition is minimal if the union of any tuple of elements of the partition is not feasible at $x$. In what follows we restrict attention only to minimal partitions. This requirement says that each resulting partition exhausts the gains of coalition formation in the sense that if the union of a tuple of elements of a compatible partition is feasible, then these elements should be joined together.

Example 1. Let $N=\{1,2,3,4\}$ and let the characteristic function $v$ of the game be given by

$$
v(S)=\left\{\begin{array}{l}
20, \text { if } S=\{i, j\}, \text { where } i \in\{1,2\} \text { and } j \in\{3,4\} \\
20, \text { if }|S|=3 \\
40, \text { if } S=N \\
0, \quad \text { otherwise }
\end{array}\right.
$$

\footnotetext{
${ }^{2}$ In the sequel of the paper, both words 'demand' and 'claim' are used.

${ }^{3}$ Notice the difference in interpretation: within the cooperative framework $x_{i}$ is a payoff, in the non-cooperative framework $x_{i}$ is a demand.

${ }^{4}$ Superscripts will refer to coalition indexing and subscripts to player indexing.
} 
Take $x=(5,10,15,10)$. Then there are four feasible coalitions, namely $\{1,3\},\{1,4\},\{2,4\}$ and $N$, resulting in six compatible partitions, namely

$$
\begin{aligned}
& \pi^{1}(x)=\{\{1\},\{2\},\{3\},\{4\}\} \\
& \pi^{2}(x)=\{\{1,3\},\{2\},\{4\}\}, \\
& \pi^{3}(x)=\{\{1,4\},\{2\},\{3\}\}, \\
& \pi^{4}(x)=\{\{2,4\},\{1\},\{3\}\}, \\
& \pi^{5}(x)=\{\{1,3\},\{2,4\}\}, \\
& \pi^{6}(x)=\{N\} .
\end{aligned}
$$

Clearly, $\pi^{6}(x)$ is the unique minimal partition. In case $v(N)=39$ and thus $N$ is not feasible anymore at $x$, only the first five partitions are compatible at $x$, while both $\pi^{3}(x)$ and $\pi^{5}(x)$ are minimal.

It should be noticed that there always exists at least one minimal partition. Clearly, this is so when there is at least one feasible coalition at $x$. If no coalition is feasible at all, the unique minimal partition is the collection of all $n$ singleton coalitions, i.e. $\pi(x)=\left\{\{i\}_{i \in N}\right\}$. For instance, this is the case for the game $(N, v)$ of Example 1 when $x=(11,11,11,11)$. Further observe that when $x(N) \leq v(N)$, the unique minimal partition is the partition in which the grand coalition $N$ forms. The next two lemmas appear to be useful in the sequel of the paper. The first one says that when $(N, v)$ is superadditive, any minimal partition contains at most one non-singleton coalition. The second one is crucial for the main result in Section 4.

Lemma 1. Let $x \in \mathbb{R}^{n}$ be a vector of demands. If the game $(N, v)$ is superadditive, then any minimal partition at $x$ is either the partition consisting of all singletons, or it has the form $\pi(x)=\left\{\{j\}_{j \in N \backslash S}, S\right\}$ for some $S \in 2^{N}$ with $|S| \geq 2$.

Proof. Clearly, when $x(S)>v(S)$, for all $S$ with $|S| \geq 2$, then $\pi(x)=$ $\left\{\{i\}_{i \in N}\right\}$ is the unique minimal partition at $x$. Otherwise, suppose $\pi(x)$ is a partition containing at least two feasible coalitions $S$ and $T$, each containing at least two players. Because of the superadditivity, then also $S \cup T$ is feasible, and this $\pi(x)$ is not minimal.

Lemma 2. Let $(N, v)$ be a superadditive game and $x \in X$ a demand vector. If $S$ (with $|S| \geq 2$ ) is a feasible coalition satisfying that also $S \cup T$ is feasible for any other feasible coalition $T$, then any minimal partition at $x$ has the form $\pi(x)=\left\{\{j\}_{j \in N \backslash S^{\prime}}, S^{\prime}\right\}$ for some $S^{\prime} \in 2^{N}$ such that $S \subseteq S^{\prime}$.

Proof. For given $x$, suppose $S$ is a coalition satisfying the above property, thus $S$ is feasible and for any $T \in 2^{N}$ it holds that $S \cup T$ is 
feasible when $T$ is feasible. Let $\pi(x)$ be a minimal partition. Since $x(S) \leq v(S), \pi(x)$ can not be the partition consisting of all singleton coalitions. When $(N, v)$ is superadditive, we know from Lemma 1 that $\pi(x)$ is of the form $\left\{\{j\}_{j \in N \backslash S^{\prime}}, S^{\prime}\right\}$ for some $S^{\prime} \in 2^{N}$ with $\left|S^{\prime}\right| \geq 2$. By definition of minimal partition, for such a coalition $S^{\prime}$ it holds that $S^{\prime}$ is feasible. Now, suppose that there is a player $j \in S \backslash S^{\prime}$. Then $\{j\}$ is a singleton coalition in $\pi(x)$. However, this contradicts the minimality of $\pi(x)$, since $S \cup S^{\prime}$ is also feasible according to the property stated for $S$. Hence, for any minimal partition $\left\{\{j\}_{j \in N \backslash S^{\prime}}, S^{\prime}\right\}$ for some $S^{\prime} \in 2^{N}$ with $\left|S^{\prime}\right| \geq 2$, we have that $S \subseteq S^{\prime}$.

We denote by $\Pi(x)$ the set of all minimal partitions at $x$ and let $\Pi_{i}(x) \subseteq \Pi(x)$ denote the set of those minimal partitions, in which player $i$ is in a coalition with at least one other player. If such a partition forms, then player $i$ is in a feasible coalition and his demand will be satisfied. When a partition not in $\Pi_{i}(x)$ forms, then player $i$ forms a coalition on its own and he will receive $v(\{i\})$. When $x_{i}>0$, then the number

$$
p_{i}(x)=\frac{\left|\Pi_{i}(x)\right|}{|\Pi(x)|}
$$

yields the probability that this positive demand will be satisfied, if we assume that all minimal partitions are equiprobable. Of course, a zero demand will always be satisfied, either within a coalition with other players or when staying alone. The function $p: X \rightarrow \mathbb{R}^{n}$, whose $i$ 's coordinate is given by (2.1), will be called the probability function. It summarizes the possibilities of the players satisfying their (positive) demands, given a specific vector of claims $x \in X$. Observe that $p_{i}(x)=$ 0 for all $i$ when $\pi(x)=\left\{\{i\}_{i \in N}\right\}$ is the unique minimal partition at $x$.

Example 2. For the game of Example 1 with $v(N)=40$ and $x=$ $(5,10,15,10)$ we have that $\pi^{6}(x)=\{N\}$ is the unique minimal partition and $p_{i}(x)=1$ for all $i \in N$. When $v(N)=39$, then $\Pi(x)=$ $\left\{\pi^{3}(x), \pi^{5}(x)\right\}$. Then $\Pi_{1}(x)=\Pi_{4}(x)=\Pi(x)$ and $\Pi_{2}(x)=\Pi_{3}(x)=$ $\left\{\pi^{5}(x)\right\}$. So, $p_{1}(x)=p_{4}(x)=1$ and $p_{2}(x)=p_{3}(x)=\frac{1}{2}$. When $x=(11,11,11,11)$, then $\Pi(x)=\left\{\pi^{1}(x)\right\}$ and $p_{j}(x)=0$ for all $j \in N$.

We are now ready to define the expected utility functions. Given a vector $x \in X$ of demands, an admissible partition $\pi(x) \in \Pi_{i}(x)$ will be realized with probability $p_{i}(x)$, consequently player $i$ receives his demand $x_{i}$. If a partition not in $\Pi_{i}(x)$ is formed, then player $i$ receives $v(\{i\})=0$. So, the expected utility (payoff) of player $i \in N$ given the vector of claims $x$ is equal to

$$
u_{i}(x)=p_{i}(x) x_{i}+\left(1-p_{i}(x)\right) v(\{i\})=p_{i}(x) x_{i} .
$$


This completes the description of the non-cooperative normal form game induced by $(N, v)$.

As standard within the non-cooperative framework, each player maximizes his expected utility given the claims (strategies) of the others. So, given the demands $x_{j}$ of all players $j$ other than $i$, denoted by $x_{-i}$, the task of player $i$ is to find a demand $x_{i}^{*}$ such that

$$
x_{i}^{*} \in \arg \max _{x_{i} \in X_{i}} p_{i}\left(x_{i}, x_{-i}\right) x_{i} .
$$

Solving this problem simultaneously for all players yields a Nash equilibrium $x^{*} \in X \subset \mathbb{R}_{+}^{n}$, satisfying the property that any deviation from $x_{i}^{*}$ to $y_{i}$ by player $i$, assuming that the other players do not change their demands, triggers a change in the probability of receiving $y_{i}$, such that $i$ 's expected utility does not increase, i.e. $x^{*}$ is such that for each player $i \in N$ and all $y_{i} \in X_{i}$ it holds that

$$
p_{i}\left(y_{i}, x_{-i}^{*}\right) y_{i} \leq p_{i}\left(x_{i}^{*}, x_{-i}^{*}\right) x_{i}^{*}
$$

where $p_{i}\left(y_{i}, x_{-i}^{*}\right)$ denotes the probability when player $i$ demands $y_{i}$ and the others stick to $x_{j}^{*}, j \in N \backslash\{i\}$. It is straightforward to see that whenever a player can increase its demand not reducing the probability with which the latter is satisfied, he will certainly do so.

Formally, define the map $\beta_{i}: \mathbb{R}_{+}^{n} \rightarrow \mathbb{R}_{+}$by

$$
\beta_{i}(x)=\left\{\widehat{x}_{i} \in \mathbb{R}_{+} \mid \widehat{x}_{i} \in \arg \max _{y_{i} \in X_{i}} p_{i}\left(y_{i}, x_{-i}\right) y_{i}\right\}
$$

Thus $\beta_{i}(x)$ gives the set of best replies of player $i$ to some vector of demands $x_{-i}$. Although the probability functions are not continuous, it is not hard to see that $\beta_{i}(x) \neq \varnothing$ for all $x$. First, observe that $p_{i}\left(0, x_{-i}\right)=0$ if and only if $x(S)>v(S \cup\{i\})$ for all non-empty $S \subseteq$ $N \backslash\{i\}$. In this case $p_{i}\left(y_{i}, x_{-i}\right)=0$ for all $y_{i} \geq 0$ and thus $\beta_{i}(x)=$ $X_{i}$. When, for given $x_{-i}$, it holds that $p_{i}\left(0, x_{-i}\right)>0$ then $p_{i}\left(y_{i}, x_{-i}\right)$ becomes a downwards step function of $y_{i}$ only, see Figure 1 for an example with $p_{i}\left(0, x_{-i}\right)=1$. 


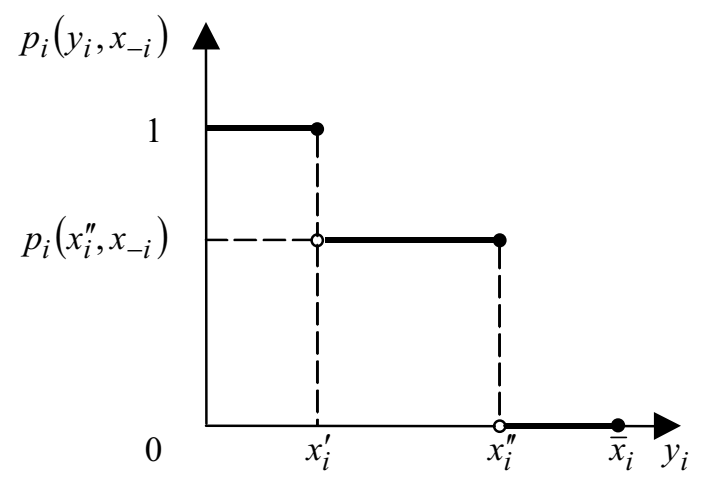

Figure 1: The probability function.

In this figure any optimal $x_{i}^{*}$ maximizes the area $p_{i}\left(y_{i}, x_{-i}\right) y_{i}$. Thus, player $i$ compares $p_{i}\left(x_{i}^{\prime}, x_{-i}\right) x_{i}^{\prime}=x_{i}^{\prime}$ with $p_{i}\left(x_{i}^{\prime \prime}, x_{-i}\right) x_{i}^{\prime \prime}$. So, although the function $p_{i}\left(y_{i}, x_{-i}\right)$ is not continuous in $y_{i}$ given $x_{-i}$, the maximization problem (2.2) always has a solution and thus $\beta_{i}(x)$ is not empty for all $x \in X$.

Next, let $\beta: \mathbb{R}_{+}^{n} \rightarrow \mathbb{R}_{+}^{n}$ be the Cartesian product of $\beta_{i}, i \in N$, for each $x \in X$, i.e.

$$
\beta(x)=\times_{i \in N} \beta_{i}(x), \text { for all } x \in X .
$$

The set of Nash equilibria of the normal form game $\left\langle N,\left\{X_{i}\right\}_{i \in N},\left\{u_{i}\right\}_{i \in N}\right\rangle$ induced by $(N, v)$ is given by the set of fixed points of $\beta$ and will be denoted by $B(N, v)$, i.e.

$$
B(N, v)=\{x \in X \mid x \in \beta(x)\} .
$$

Hence, any vector $x \in B(N, v)$ describes a stable social situation (or a mode of behavior). Such vectors will be called stable demands and correspondingly $B(N, v)$ will be called the stable demand set. It is convenient to think of any vector $x \in B(N, v)$ as being a convention in the spirit of Young [16]. That is, an arrangement such that no player can individually gain by not following the convention. This interpretation of the set $B(N, v)$ is close to the notion of von NeumannMorgenstern (henceforth vNM) stable set. For convex games we will show that this interpretation is indeed correct. However, in general the two concepts may be quite different as will be demonstrated by the Examples 4 and 5 in Section 6.1.

Applying the notion of strict Nash equilibria (in which each player $i$ has a unique best reply to the profile of strategies $x_{-i}$ ) for the above set $B(N, v)$ leads to the following definition

$$
\widetilde{B}(N, v)=\{x \in X \mid\{x\}=\beta(x)\} .
$$


The set $\widetilde{B}(N, v)$ is called the strict stable demand set. Obviously, $\widetilde{B}(N, v) \subseteq B(N, v)$ for any game $(N, v)$. We remark that although it is not easy to find all stable demands in arbitrary games, it is relatively simple to check if a specific vector of claims is (strict) stable. Most of the applications discussed below will evolve around checking this condition.

Before we consider the properties of the (strict) stable demand set, we first shortly discuss the related literature on demand adjustment.

\section{Related Literature}

One of the earliest papers dealing with demand adjustment is Cross [10]. It models a competitive process, in which players set their prices for participation in different coalitions. The process develops in such a way that the payoff of scarce players (whose participation is needed in at least two coalitions) is driven up, which, in turn, reduces the surplus available to the others. The set of stable prices can be shown to be identical to the set of balanced aspirations, see Bennett [3].

Bennett et al. [6] considers a game, in which players adjust their demands based on the availability of coalitions that support these demands. Specifically, it is assumed that

i) only one player adjusts at a time.

ii) a player will increase his demand if some coalition can support the larger demand, given the demands of others.

iii) a player will decrease his demand if no coalition can support his current demand, given the demands of others.

Theorem 1 in [6] characterizes conditions, under which the above adjustment process converges to an aspiration regardless of the initial vector of demands. Observe that while adjusting their demands, the players are governed exclusively by maximality and feasibility of their aspirations. They do not take into account the probability of their demands being satisfied.

The current paper is much in the spirit of Agastya [1]. Theorem 1 in [1] shows that if the underlying game $(N, v)$ is convex then an allocation $x$ can become a convention in the language of Young [16] (in our terminology strict stable demand) if and only if $x$ belongs to the discrete core of $(N, v)$, where discretization comes from the assumption that there is a smallest money unit $\epsilon$ by which players can adjust their demands. This result was obtained for any probability function $p$, satisfying the following two properties

i) if for a given demand vector $x$, there is no feasible coalition containing $i$, then $p_{i}(x)=0$. 
ii) if $S$ (with $|S| \geq 2$ ) is a feasible coalition at demand vector $x$ and also $S \cup T$ is feasible at $x$ for any other feasible coalition $T$, then $p_{i}(x)=1$ for all $i \in S$.

In case the game $(N, v)$ is superadditive, our probability function as defined in equation (2.1) satisfies both conditions. First, when no feasible coalition contains player $i$, then player $i$ is a singleton coalition in any minimal partition and thus $p_{i}(x)=0$ (property (i)). Second, let $S$ be a coalition satisfying property (ii), then $S$ satisfies the property of Lemma 2 and thus it follows from that lemma that any minimal partition is of the form $\left\{\{j\}_{j \in N \backslash S^{\prime}}, S^{\prime}\right\}$ for some $S^{\prime} \in 2^{N}$ such that $S \subseteq S^{\prime}$, implying that $p_{i}(x)=1$ for all $i \in S$. This yields that for superadditive games our probability function is a specific choice within the class of functions allowed by Agastya. In this light our approach is more restrictive. On the other hand, in the sequel of this paper we will also consider non-convex and even not superadditive games. Moreover, the specific form of the probability function we employ appears to be quite natural for certain classes of games, like market games, and also enables us to derive a number of explicit results. These results show that the solutions induced by the probabilities as defined by (2.1) may highlight interesting conventions that have not been captured by existing solution concepts. The further important difference between our setup and that of Agastya [1] is that we do not introduce the smallest money unit by which demands can be changed. This assumption may lead to the non-existence of conventions, see Example 3 in [1]. In Section 5.1 we will reconsider this example and show that strict stable demands do exist when applying our probability function.

\section{Properties of Stable Demands}

We will be mainly interested in the properties of the stable demand set $B(N, v)$, and specifically in the conditions, under which the strict stable demand set is non-empty. In order to establish non-emptiness, however, one cannot apply usual fixed point theorems. The reason is that $\beta_{i}(x)$ is not continuous in $x$ because of the discontinuity of the probability functions. Small changes in $x$ may change the cardinality of the sets $\Pi(x)$ and $\Pi_{i}(x)$ and therefore cause jumps in the probabilities. Nevertheless, a little reflection shows that $B(N, v)$ is non-empty for any game by the following argument. Take $x \in \mathbb{R}^{n}$ such that $x_{i}=\bar{x}_{i}$ for all $i$. Then $\Pi(x)=\left\{\{i\}_{i \in N}\right\}$, since $\bar{x}_{i}>v(S)$ for all $S \in 2^{N}$. Consequently $\Pi_{i}(x)=\varnothing$, so that $p_{i}(x)=0$ and $\beta_{i}(x)=X_{i}$ for all $i \in N$. Hence $x \in \beta(x)$, so the vector of demands $x=\bar{x}$ is trivially stable. This holds for any vector $x$ such that $x(S)>v(S)$ for all $S$ 
satisfying $|S| \geq 2$. Of course such stable demands are degenerate and naturally one would be interested whether there are 'more sensible' demand vectors that are stable. The following proposition shows that this is indeed the case.

Proposition 1. For any game $(N, v)$ it holds that $C(N, v) \subseteq B(N, v)$ and $\widetilde{C}(N, v) \subseteq \widetilde{B}(N, v)$.

Proof. Take a vector of demands $x \in \widetilde{C}(N, v)$. Then $x(N) \leq v(N)$ and $\pi(x)=\{N\}$ is the unique minimal partition compatible with $x$, so that all players' demands are satisfied with probability one. Clearly for any player $i \in N$ it is not profitable to decrease demand, since this will not improve $i$ 's expected utility under any circumstances. Moreover, increasing demand will leave no coalition that satisfies $i$ 's claim because of the condition $x(S) \geq v(S)$ for all $S \in 2^{N}$. Hence, no player $i \in N$ has a profitable deviation from $x \in \widetilde{C}(N, v)$ and since $x_{i}>0$ for all $i \in N$, the vector $x$ is strict stable. For any $x \in C(N, v) \backslash \widetilde{C}(N, v)$, by the above reasoning no player, $i \in N$ has a profitable deviation either, however strict stability is violated, since for at least one $i$ we have $x_{i}=0$.

Proposition 1 implies that the sufficient condition for the non-emptiness of $\widetilde{B}(N, v)$ is the non-emptiness of $\widetilde{C}(N, v)$. The latter set may be empty even if $C(N, v)$ is not, since the core may consist only of allocations $x$ with $x_{i}=0$ for some $i \in N$. However, $\widetilde{C}(N, v)$ is always nonempty for convex games satisfying the mild condition that the game $(N, v)$ does not have null players. ${ }^{5}$ To see why this is so, let $\Sigma(N)$ be the set of permutations $\sigma: N \rightarrow N$. For a permutation $\sigma \in \Sigma(N)$ denote by $m_{i}^{\sigma}$ the marginal contribution of player $i \in N$ as

$$
m_{i}^{\sigma}=v(\{j \in N \mid \sigma(j) \leq \sigma(i)\})-v(\{j \in N \mid \sigma(j)<\sigma(i)\}) .
$$

It is well-known that the core of a convex game is the convex hull of the marginal vectors. If for every $i \in N$ there is at least one $\sigma$, such that $m_{i}^{\sigma}>0$, i.e. no player is null, then there exists a vector of demands $x \in C(N, v)$, such that $x_{i}>0$.

We now present three useful lemmas that characterize the set $\widetilde{B}(N, v)$ for the class of convex TU-games. The first lemma holds for any game and states that for every stable demand vector $x$ the total demand $x(N)$ is at least equal to the worth of the grand coalition.

Lemma 3. Let $x \in B(N, v)$ be a stable demand vector. Then $x(N) \geq$ $v(N)$.

\footnotetext{
${ }^{5}$ A player $i \in N$ is a null player if for all $S \subseteq N$ it holds that $v(S \cup\{i\})=v(S)$.
} 
Proof. Suppose $x(N)<v(N)$. Then the unique minimal partition is $\pi(x)=\{N\}$ and thus $p_{i}(x)=1$ for all $i \in N$. Hence, any player $i$ can increase his demand to $y_{i}=v(N)-\sum_{j \in N \backslash\{i\}} x_{j}$ and still get it satisfied with probability one. This contradicts that $x$ is stable.

The next two lemmas hold for convex games. Their proofs are similar to the corresponding proofs in Agastya [1]. For the proof of the next lemma, in Agastya's model it is essential that the probability function satisfies property (ii) as discussed in the previous section. For our specific choice of the probability function, we can use Lemma 2 directly.

Lemma 4. Let $(N, v)$ be a convex game and $x \in B(N, v)$. Then $x(S) \geq v(S)$ for all $S \in 2^{N}$.

Proof. Suppose there is a coalition, say $S^{1}$, such that $x\left(S^{1}\right)<v\left(S^{1}\right)$. We show that then there is a player $i \in S^{1}$, who can increase his demand and get this increased demand with probability one; hence such a vector of claims $x$ cannot be stable. Take some $i \in S^{1}$ and vector $y^{1} \in X$ given by

$$
\begin{aligned}
& y_{i}^{1}=v\left(S^{1}\right)-\sum_{j \in S^{1} \backslash\{i\}} x_{j}>x_{i}, \\
& y_{j}^{1}=x_{j} \text { for all } j \in N \backslash\{i\} .
\end{aligned}
$$

If coalition $S^{1}$ is feasible at $y$ and satisfies the property of Lemma 2, then that lemma immediately implies the desired result that $p_{i}\left(y^{1}\right)=1$. Next, consider the case that there is a feasible coalition $T$ at $y^{1}$, but that $S^{1} \cup T$ is not feasible at $y^{1}$, thus $y^{1}\left(S^{1} \cup T\right)>v\left(S^{1} \cup T\right)$. Observe that $S^{1} \cap T$ is non-empty, since $S^{1}$ and $T$ are both feasible and the game is convex and thus superadditive, and that $\left|S^{1} \cap T\right|<\left|S^{1}\right|$ because otherwise $S^{1} \subseteq T$, which contradicts that $S^{1} \cup T$ is not feasible. Using the identity $y^{1}\left(S^{1} \cup T\right)=y^{1}\left(S^{1}\right)+y^{1}(T)-y^{1}\left(S^{1} \cap T\right)$, that $S^{1}$ and $T$ are feasible at $y^{1}$ and that $S^{1} \cup T$ is not, it follows from the convexity of $(N, v)$ that $y^{1}\left(S^{1} \cap T\right)<v\left(S^{1} \cap T\right)$. Then define $S^{2}:=S^{1} \cap T$ and repeat the same reasoning as for $S^{1}$. If needed, subsequently define $S^{3}$, $S^{4}$, etc. In $k \leq\left|S^{1}\right|-2$ steps a set $S^{k}$ with $\left|S^{k}\right| \geq 2$ is reached and a vector $y^{k}$ with respect to some $i \in S^{k} \subseteq S^{1}$, such that $S^{k}$ is feasible at $y^{k}$ and satisfies the property of Lemma 2 . This is so, because otherwise in $m \leq\left|S^{1}\right|-1$ steps the procedure would result in a single player set $S^{m}=\{j\}$ for some $j \in S^{1}$, and $y_{j}^{m}<v(\{j\})$, which contradicts that $y_{j}^{m} \geq x_{j} \geq 0=v(\{j\})$. So, in a finite number of steps we obtain a player in $S^{1}$ that can increase his demand and get this increased demand with probability one.

Lemma 5. Let $(N, v)$ be a convex game and let $x \in \widetilde{B}(N, v)$. Then $x(N)=v(N)$. 
Proof. A necessary condition for $x$ to be in the strict stable demand set is that for all $i \in N$ we have both $x_{i}>0$ and $p_{i}(x)>0$. From the latter condition it follows that for each $i$ there exists a coalition $S^{i}$ such that $x\left(S^{i}\right) \leq v\left(S^{i}\right)$. Take a vector of claims $x$ and consider two different players $i$ and $j$ with corresponding coalitions $S^{i}$ and $S^{j}$. Using the convexity of $(N, v)$ and Lemma 4, it follows straightforward that $x\left(S^{i} \cup S^{j}\right) \leq v\left(S^{i} \cup S^{j}\right)$. Generalizing this reasoning for all players $i \in N$ we obtain that $x(N) \leq v(N)$. Together with Lemma 3 it follows that $x(N)=v(N)$.

We are now ready to state the main result of this section, which is the modification of Theorem 1 in Agastya [1] adapted to our specific form of the probability function and choice of the strategy space.

Theorem 1. Let $(N, v)$ be a convex game. Then $\widetilde{B}(N, v) \equiv \widetilde{C}(N, v)$.

Proof. The inclusion $\widetilde{C}(N, v) \subseteq \widetilde{B}(N, v)$ follows from Proposition 1 . The other inclusion $\widetilde{C}(N, v) \supseteq \widetilde{B}(N, v)$ is a consequence of Lemmas 4 and 5 , combined with the fact that $\widetilde{B}(N, v) \subseteq B(N, v)$.

It is well-known that a convex game has a unique vNM stable set which coincides with the core. Thus the above theorem shows that for convex games the set of strict best replies $\widetilde{B}(N, v)$ coincides with the set of allocations in the vNM stable set, satisfying $x_{i}>0$ for all $i \in N$. If the underlying game is not convex, this relation between $\widetilde{B}(N, v)$ and the stable set is lost. The equivalence between $\widetilde{C}(N, v)$ and $\widetilde{B}(N, v)$ also implies the convexity of $\widetilde{B}(N, v)$ when $(N, v)$ is convex. Thus for the class of convex games the set of extreme points of the closure of $\widetilde{B}(N, v)$ can be easily characterized. Consequently one can find how large (in the limit) the demand $x_{i}$ of a particular player $i \in N$ can be, so that $x$ is a convention. In general, the set $\widetilde{B}(N, v)$ does not have to be convex, see Section 6.2 for an example.

We conclude this section with the interesting question under what conditions strict stable demands are efficient, i.e. the demands sum up to the worth of the grand coalition. From the above theorem it is clear that for convex games this is always the case. Moreover, we then have that the unique minimal partition is the partition in which the grand coalition forms and thus $p_{i}(x)=1$ for all $i \in N$, implying that any player gets his demand with certainty and thus also the total (expected) payoff is equal to $v(N)$.

For non-convex games the demands sum up to at least $v(N)$, as shown in Proposition 1. In such cases it makes sense to see whether a given strict stable demand vector $x$ is efficient in its expected form, i.e. 
whether $\sum_{i \in N} x_{i} p_{i}(x)=v(N)$ holds. For arbitrary games this question is hard to answer. However if $(N, v)$ is superadditive, the characterization can be stated in a rather simple way, as the following proposition demonstrates. In what follows we denote $E[x]=\sum_{i \in N} x_{i} p_{i}(x)$.

Proposition 2. Let $(N, v)$ be a superadditive game. Then for any $x \in \widetilde{B}(N, v)$ we have $E[x] \leq v(N)$. Moreover this holds with equality if and only if for any feasible coalition $S$ at $x$, which belongs to some minimal partition $\pi \in \Pi(x)$, it holds that $x(S)=v(N)$.

Proof. Take $x \in \widetilde{B}(N, v)$. As $(N, v)$ is superadditive, each $\pi^{k} \in \Pi(x)$ contains exactly one feasible coalition $S^{k}$ by Lemma 1 (it cannot contain only singletons, since then $x \in \widetilde{B}(N, v)$ is violated). Thus the total number of all such coalitions is $|\Pi(x)|$. Let $|\Pi(x)|=m$, then

$$
E[x]=\frac{\sum_{i \in N} \sum_{\left\{S^{k} \mid i \in S^{k}\right\}} x_{i}}{m}=\frac{\sum_{k=1}^{m} x\left(S^{k}\right)}{m} \leq \frac{m v(N)}{m} \leq v(N),
$$

since by superadditivity $v(S) \leq v(N)$ for all $S \in 2^{N}$. Furthermore, if for each $S^{k}$, where $k=1, \ldots, m$ one has $x\left(S^{k}\right)=v(N)$, then the above condition holds with equality, as required.

Note also that if there is no any feasible coalition at $x$, then the unique minimal partition is $\pi(x)=\left\{\{j\}_{j \in N}\right\}$ and clearly $x \notin \widetilde{B}(N, v)$.

Proposition 2 tells us that for superadditive games inefficiencies may easily arise, namely when a minimal partition is realized for which the worth of the unique feasible coalition $S$ in the partition is below the worth of the grand coalition. The latter naturally arises in such superadditive games as market games, see Section 6.1. Unfortunately, Proposition 2 does not extend to games that are not superadditive, as is easy to show by an example.

\section{Three-Player Games with Empty Cores}

Our focus in this section is to find non-trivial demand vectors that are (strict) stable. For games with non-empty cores such demands are described in Proposition 1. Here we consider three-player games with empty cores and, in particular, investigate the stability of a popular solution concept for this class of games - the vNM vector. Again, throughout this section it is assumed that the game is zero-normalized and thus $v(\{i\})=0$ for all $i \in N$. For ease of notation, in the following we will write the characteristic function values of any two-player coalition as $v(\{j, k\})=v(j k)$. Moreover, for $i \in\{1,2,3\}$, we denote the two other players by $j$ and $k$, thus $\{j, k\}=\{1,2,3\} \backslash\{i\}$. 
We start with defining the vNM vector for this class of three-player games. Consider the following system of equations

$$
\left\{\begin{array}{l}
x_{1}+x_{2}=v(12), \\
x_{1}+x_{3}=v(13), \\
x_{2}+x_{3}=v(23) .
\end{array}\right.
$$

Clearly, it has a unique solution, say $z=\left(z_{1}, z_{2}, z_{3}\right)$, given by

$$
z_{i}=\frac{v(i j)+v(i k)-v(j k)}{2}, i=1,2,3 .
$$

Observe that by definition, $z_{1}+z_{2}+z_{3}=\frac{1}{2}[v(12)+v(13)+v(23)]$. So, when $v(12)+v(13)+v(23)<2 v(N)$, then $z_{1}+z_{2}+z_{3}<v(N)$. In this case, the game is balanced and thus has a non-empty core, but the vNM vector is not defined. When $v(12)+v(13)+v(23) \geq 2 v(N)$ and thus $z_{1}+z_{2}+z_{3} \geq v(N)$, then according to Binmore [8], $z$ is defined to be the vNM vector, provided $z_{i}, z_{j}, z_{k} \geq 0$. Note that if $z_{1}+z_{2}+z_{3}=v(N)$, the game is balanced and it follows straightforward that $z$ is the unique element in the core and thus, according to Proposition 1, a stable demand vector (and strict stable when $z_{i}>0$ for all $i \in N$ ).

5.1. Superadditive games. Suppose we are given a superadditive three-player game with empty core, thus $v(N) \geq v(j, k)$ for any twoplayer coalition $\{j, k\} \subset\{1,2,3\}$ and $z_{1}+z_{2}+z_{3}=\frac{1}{2}[v(12)+v(13)+$ $v(23)]>v(N)$. It then follows that $z_{i}>v(N)-z_{j}-z_{k}=v(N)-v(j k) \geq$ 0 for all $i=1,2,3$ and thus $z$ is defined as the vNM vector. The following result says that $z$ is a stable demand vector when the value of each two-player coalition is high enough.

Proposition 3. Let $(N, v)$ be a superadditive three-player game with an empty core and let $z$ be its $v N M$ vector. Then $z \in B(N, v)$ if and only if

$$
v(12)+v(13)+v(23)+v(j k) \geq 3 v(N), \text { for each pair }\{j, k\},
$$

with $z \in \widetilde{B}(N, v)$ when this condition holds with strict inequality.

Proof. Since $z_{1}+z_{2}+z_{3}=\frac{1}{2}[v(12)+v(13)+v(23)]>v(N)$ because the assumption that the core is empty, and $z_{j}+z_{k}=v(j k)$, the set of minimal partitions at the demand vector $z$ is given by

$$
\Pi(z)=\{\pi(z) \mid \pi(z)=\{\{i\},\{j, k\}\}\},
$$

thus only two-player coalitions can form given $z$. Consequently, $p_{i}(z)=$ $\frac{2}{3}$ for all $i \in N$. Now, suppose that $i$ has a profitable deviation from $z_{i}$. Such a deviation necessarily involves a reduce of the demand, since an increase will leave no coalition in which player $i$ can participate because 
of $z_{i}+z_{j}=v(i j)$ for $j \neq i$. Furthermore, reducing demand is profitable only when $i$ reduces it to $x_{i}=v(N)-v(j k)$, so that the unique minimal partition will be the grand coalition and thus $p_{i}\left(x_{i}, z_{j}, z_{k}\right)=1$. Hence, $z_{i}$ is a best reply of player $i$ to $z_{j}$ and $z_{k}$ if and only if

$$
\frac{2}{3} z_{i} \geq v(N)-v(j k)
$$

while it is the unique best reply when this holds with strict inequality. Using $z_{i}=\frac{v(i j)+v(i k)-v(j k)}{2}$, this yields the condition

$$
v(i j)+v(i k)-v(j k) \geq 3 v(N)-3 v(j k) .
$$

So, $z$ is a stable demand vector iff

$$
v(i j)+v(i k)+2 v(j k) \geq 3 v(N), \text { for all } i=1,2,3,
$$

which yields the condition (5.1). Further, for every player $i, z_{i}$ is the unique best reply and thus $z$ is strict stable when this holds with strict inequality for all $i=1,2,3$

If the $\mathrm{vNM}$ vector $z$ is stable but not strict, there may exist other stable demands that are strict. See Example 3 below for an illustration.

In what follows we will characterize some other (strict) stable demand vectors in three-player superadditive games. First, note that Proposition 3 does not cover all cases with $z_{1}+z_{2}+z_{3}>v(N)$. For example, when $v(12)=v(13)=v(23)$, then $z_{1}+z_{2}+z_{3}=\frac{3}{2} v(12)>v(N)$ holds if $v(12)>\frac{2}{3} v(N)$, while the condition (5.1) in Proposition 3 reduces to $v(12) \geq \frac{3}{4} v(N)$. So, in case all two-player coalitions have equal value $v(12)$, neither Proposition 1 nor Proposition 3 provides the existence of a stable demand vector when

$$
\frac{2}{3} v(N)<v(12)<\frac{3}{4} v(N) .
$$

Similarly, when $v(N)=v(12)=v(13) \geq v(23)$, then $z_{1}+z_{2}+z_{3}=$ $\frac{1}{2}[v(12)+v(13)+v(23)]=v(N)+\frac{1}{2} v(23)>v(N)$ is satisfied for any $v(23)>0$, while condition (5.1) in Proposition 3 reduces to $v(23) \geq$ $\frac{1}{2} v(N)$. So, in this case neither Proposition 1 nor Proposition 3 provides the existence of a stable demand vector when

$$
0<v(23)<\frac{1}{2} v(N) .
$$

The proposition below gives a set of stable demand vectors for any set of values satisfying neither the condition of Proposition 1 (nonemptiness of the core) nor condition (5.1) of Proposition 3. To state this result, define $M_{i}=v(N)-v(j k), i=1,2,3$. Clearly, $M_{i} \geq 0$ because of the superadditivity of $(N, v)$. Further, when $C(N, v)=\varnothing$ it 
follows $v(12)+v(13)+v(23)>2 v(N)$. Moreover, for all $i \in N$ it holds that

$$
M_{i}=v(N)-v(j k)<\frac{1}{2}[v(i j)+v(i k)-v(j k)]=z_{i} .
$$

Finally, observe that when the condition (5.1) of Proposition 3 does not hold, then there exists at least one $i$ such that $v(i j)+v(i k)+2 v(j k)<$ $3 v(N)$ and thus $z_{i}<\frac{3}{2}[v(N)-v(j k)]=\frac{3}{2} M_{i}$. Define

$$
K(N, v)=\left\{i \in\{1,2,3\} \mid z_{i}<\frac{3}{2} M_{i}\right\}
$$

and let $W(N, v)$ be given by

$$
W(N, v)=\operatorname{Conv}\left\{y^{i} \mid i \in K(N, v)\right\},
$$

where Conv denotes the convex hull and where $y^{i} \in \mathbb{R}^{n}$ is given by $y_{i}^{i}=M_{i}$ and $y_{j}^{i}=z_{j}, j \neq i$. As shown above, $W(N, v)$ is non-empty when condition (5.1) of Proposition 3 is not satisfied. We now have the following result.

Proposition 4. Let $(N, v)$ be a superadditive three-player game with empty core, such that

$$
v(i j)+v(i k)+2 v(j k)<3 v(N), \text { for at least one } i \in N .
$$

Then $W(N, v) \subseteq B(N, v)$, with $W(N, v) \subseteq \widetilde{B}(N, v)$ when $v(j k)<v(N)$ for any pair $\{j, k\}$.

Proof. We consider three cases, namely $K(N, v)$ contains one, two or three players. Observe that $i \in K(N, v)$ implies that $M_{i}>0$ and thus $v(j k)<v(N)$.

Case (i). $|K(N, v)|=1$. Without loss of generality we take $K(N, v)=$ $\{1\}$, so that we have to show that $y^{1}$ is stable. First, since $\sum_{j} y_{j}^{1}=$ $M_{1}+z_{2}+z_{3}=M_{1}+v(23)=v(N)$, we have that $p_{j}\left(y^{1}\right)=1$ for all $j=1,2,3$ and thus the expected utility is equal to $y_{j}^{1}$ for all $j$. Clearly any deviation in which a player decreases his demand yields a lower expected utility, so only those deviations in which players increase their demands have to be considered.

Suppose that $j=1$ deviates by demanding $x_{1}>M_{1}$. Then the grand coalition can not be formed any more and player 1 will receive any demand $x_{1}$ with $M_{1}<x_{1} \leq z_{1}$ with probability $\frac{2}{3}$ and any demand $x_{1}>z_{1}$ with probability zero. However, since $1 \in K(N, v)$, we have that $\frac{2}{3} z_{1}<M_{1}$ and thus such a deviation $x_{1}>M_{1}$ is not profitable. Hence $M_{1}$ is the unique best reply of player 1 . For player $j \neq 1$, observe that $v(1 j)-M_{1}>v(1 j)-z_{1}$, since $M_{1}<z_{1}$ because of the fact that 
the core is empty. So, when a player $j \neq 1$ deviates from $y^{1}$ and states a demand $x_{j}$ such that $y_{j}^{1}=z_{j}<x_{j} \leq v(1 j)-M_{1}$, we have that

$$
\begin{aligned}
x_{j}+y_{1}^{1} & =x_{j}+M_{1} \leq v(1 j), \\
x_{j}+y_{k}^{1} & =x_{j}+z_{k}>v(j k), \\
y_{1}^{1}+y_{k}^{1} & =M_{1}+z_{k}<v(1 k), \\
y_{1}^{1}+x_{j}+y_{k}^{1} & =M_{1}+x_{j}+z_{k}>v(N),
\end{aligned}
$$

and thus player $j$ receives this demand with probability $\frac{1}{2}$. Moreover, $j$ will receive his demand $x_{j}$ with probability zero when $x_{j}>v(1 j)-M_{1}$. Therefore $y_{j}^{1}=z_{j}$ is a best reply of $j$ if

$$
\frac{1}{2}\left[v(1 j)-M_{1}\right] \leq z_{j}
$$

thus if

$$
\frac{v(1 j)-(v(N)-v(j k))}{2} \leq \frac{v(1 j)+v(j k)-v(1 k)}{2}
$$

Clearly this reduces to $v(1 k) \leq v(N)$ for $k \neq 1, j$, which holds because of the superadditivity. So, $y_{j}^{1}=z_{j} \in \beta_{j}\left(y^{1}\right)$ and it is the unique best reply when $v(1 k)<v(N)$. This proves the proposition when $|K(N, v)|=1$.

Case (ii). $|K(N, v)|=2$. Without loss of generality we take $K(N, v)=$ $\{1,2\}$, so that $W(N, v)=\operatorname{Conv}\left\{y^{1}, y^{2}\right\}$. Let $y \in W(N, v)$. When $y=y^{1}$ or $y=y^{2}$, the proof that $y$ is stable is identical to case (i). For any other $y$, we have that

$$
y=\lambda y^{1}+(1-\lambda) y^{2}, \text { for some } 0<\lambda<1
$$

So,

$$
\begin{aligned}
M_{1}<y_{1} & =\lambda M_{1}+(1-\lambda) z_{1}<z_{1} \\
M_{2}<y_{2} & =\lambda z_{2}+(1-\lambda) M_{2}<z_{2}, \\
y_{3} & =\lambda z_{3}+(1-\lambda) z_{3}=z_{3} .
\end{aligned}
$$

Further $\sum_{j} y_{j}=\lambda \sum_{j} y_{j}^{1}+\sum_{j}(1-\lambda) y_{j}^{2}=\lambda v(N)+(1-\lambda) v(N)=v(N)$. Thus $p_{j}(y)=1$ and each player gets his demand $y_{j}$ with certainty, so that only deviations in which a player increases his demand have to be considered. 
We first show that $y_{1} \in \beta_{1}(y)$. When player 1 increases his demand to $z_{1}$, then

$$
\begin{aligned}
z_{1}+y_{2}+y_{3} & >v(N), \\
z_{1}+y_{2} & <z_{1}+z_{2}=v(12), \\
z_{1}+y_{3} & =z_{1}+z_{3}=v(13), \\
y_{2}+y_{3} & <z_{2}+z_{3}=v(23),
\end{aligned}
$$

so that player 1 will receive demand $z_{1}$ with probability $\frac{2}{3}$. However, since $1 \in K(N, v), \frac{2}{3} z_{1}<M_{1}<y_{1}$, so that any demand $x_{1}$ satisfying $y_{1}<x_{1} \leq z_{1}$ is not a best reply of player 1 to $y$. When player 1 further increases his demand to $\widetilde{z}_{1}=v(12)-y_{2}$, then

$$
\begin{aligned}
\widetilde{z}_{1}+y_{2}+y_{3} & >v(N), \\
\widetilde{z}_{1}+y_{2} & =v(12), \\
\widetilde{z}_{1}+y_{3} & >z_{1}+z_{3}=v(13), \\
y_{2}+y_{3} & <z_{2}+z_{3}=v(23),
\end{aligned}
$$

so that player 1 will receive $\widetilde{z}_{1}$ with probability $\frac{1}{2}$. So, $y_{1}$ is at least as $\operatorname{good}$ as $\widetilde{z}_{1}$ if

$$
\frac{1}{2} \widetilde{z}_{1}=\frac{1}{2}\left[v(12)-y_{2}\right] \leq y_{1}
$$

thus if

$$
v(12)-\lambda z_{2}-(1-\lambda) M_{2} \leq 2 y_{1}=2\left[\lambda M_{1}+(1-\lambda) z_{1}\right] .
$$

Observe that both the left-hand side and the right-hand side are linear in $\lambda$. Define, for $\lambda \in[0,1]$,

$$
f(\lambda)=v(12)-\lambda z_{2}-(1-\lambda) M_{2} \text { and } g(\lambda)=2\left[\lambda M_{1}+(1-\lambda) z_{1}\right] .
$$

Then $f(1)=v(12)-z_{2}=z_{1}$ and $g(1)=2 M_{1}$, so that $f(1)<g(1)$, since $z_{1}<\frac{3}{2} M_{1}$. On the other hand $f(0)=v(12)-M_{2}=v(12)+v(13)-v(N)$ and $g(0)=2 z_{1}=v(12)+v(13)-v(23)$. So, $f(0) \leq g(0)$ because of the superadditivity. Because of the linearity it follows that $f(\lambda)<g(\lambda)$ for all $0<\lambda<1$ and thus $y_{1}$ gives higher expected utility than any demand $x_{1}$ satisfying $z_{1}<x_{1} \leq \widetilde{z}_{1}$. Further, player 1 can not form any coalition when he demands $x_{1}>\widetilde{z}_{1}$. So, for any $0<\lambda<1, y_{1}$ is the unique element of $\beta_{1}(y)$. Analogously it follows that $y_{2}$ is the unique best reply of player 2 . 
It remains to prove that $y_{3} \in \beta_{3}(y)$. Recall that

$$
\begin{aligned}
& y_{1}+y_{3}=y_{1}+z_{3}<v(13) \\
& y_{2}+y_{3}=y_{2}+z_{3}<v(23) \\
& y_{1}+y_{2}<z_{1}+z_{2}=v(12)
\end{aligned}
$$

Further, recall that $y=\lambda y^{1}+(1-\lambda) y^{2}$. Therefore $v(13)-y_{1}=v(23)-y_{2}$ when

$$
v(13)-\left[\lambda M_{1}+(1-\lambda) z_{1}\right]=v(23)-\left[\lambda z_{2}+(1-\lambda) M_{2}\right] .
$$

Solving for $\lambda$ yields $\lambda=\frac{1}{2}$, with $v(13)-y_{1} \leq v(23)-y_{2}$ iff $\lambda \leq \frac{1}{2}$. First, consider $\lambda \leq \frac{1}{2}$ and suppose that player 3 increases his demand to $\widetilde{z}_{3}=\min \left\{v(13)-y_{1}, v(23)-y_{2}\right\}=v(13)-y_{1}$. Then any two-player coalition can still fulfill their demands, so that player 3 receives this demand with probability $\frac{2}{3}$. So, $y_{3}=z_{3}$ is at least as good as $\widetilde{z}_{3}$ if

$$
\frac{2}{3} \widetilde{z}_{3}=\frac{2}{3}\left[v(13)-y_{1}\right] \leq z_{3} \text {. }
$$

Since $y_{1}=\lambda M_{1}+(1-\lambda) z_{1} \geq \frac{1}{2}\left(M_{1}+z_{1}\right)$ for all $\lambda \leq \frac{1}{2}$ (with strict inequality when $\lambda<\frac{1}{2}$ ), it follows that $\frac{2}{3} \widetilde{z}_{3} \leq z_{3}$ if

$$
\frac{2}{3}\left[v(13)-\frac{1}{2} M_{1}-\frac{1}{2} z_{1}\right] \leq z_{3}
$$

which again reduces to $v(12) \leq v(N)$ and therefore holds because of the superadditivity. So, $z_{3}$ is at least as good as $\widetilde{z}_{3}$ and strictly better if $v(12)<v(N)$ or if $\lambda<\frac{1}{2}$. Analogously the case $\lambda \geq \frac{1}{2}$ and $\widetilde{z}_{3}=$ $\min \left\{v(13)-y_{1}, v(23)-y_{2}\right\}=v(23)-y_{2}$ follows.

Next, consider $\lambda<\frac{1}{2}$ and suppose that player 3 increases his demand to $\widehat{z}_{3}=\max \left\{v(13)-y_{1}, v(23)-y_{2}\right\}=v(23)-y_{2}>v(13)-y_{1}$. Then, only the coalitions $\{2,3\}$ and $\{1,2\}$ can fulfill their demands, so that player 3 gets this demand with probability $\frac{1}{2}$. So, $y_{3}=z_{3}$ is at least as $\operatorname{good}$ as $\widehat{z}_{3}$ if

$$
\frac{1}{2} \widehat{z}_{3}=\frac{1}{2}\left[v(23)-y_{2}\right] \leq z_{3} .
$$

Since $y_{2}>M_{2}$ this is true (with strict inequality) if

$$
\frac{1}{2}\left[v(23)-M_{2}\right] \leq z_{3}
$$

which again reduces to $v(12) \leq v(N)$ and therefore holds because of the superadditivity. So, $z_{3}$ is strictly better than $\widehat{z}_{3}$. Analogously the case $\lambda>\frac{1}{2}$ and $\widehat{z}_{3}=\max \left\{v(13)-y_{1}, v(23)-y_{2}\right\}=v(13)-y_{1}$ follows. Clearly, player 3 can not form any coalition when he demands $x_{3}>\widehat{z}_{3}$. 
So, for any $0<\lambda<1, y_{3} \in \beta_{3}(y)$ and it is the unique best reply when $v(12)<v(N)\left(\right.$ or $\left.\lambda \neq \frac{1}{2}\right)$. This proves case (ii).

Case (iii). $K(N, v)=\{1,2,3\}$. First recall that in this case $0<$ $M_{i}<z_{i}<\frac{3}{2} M_{i}$ for all $i$ and thus $v(j k)<v(N)$ and $v(12)+v(13)+$ $v(23)+v(j k)<3 v(N)$ for any pair $\{j, k\}$. Let $y \in W(N, v)$. When $y=y^{i}$ for some $i$, the proof that $y$ is stable is identical to case (i). Also, when $y$ is a convex combination of $y^{i}$ and $y^{j}$ for some $i$ and $j$, the proof is identical to case (ii). Otherwise,

$$
y=\lambda_{1} y^{1}+\lambda_{2} y^{2}+\lambda_{3} y^{3}
$$

with $\lambda_{j}>0$ for all $j$ and $\sum_{j} \lambda_{j}=1$. We show that $y_{3} \in \beta_{3}(y)$. Without loss of generality we suppose that $v(13)-y_{1} \leq v(23)-y_{2}$. Clearly, $y_{1}+y_{2}+y_{3}=v(N)$ because $y$ is a convex combination of $y^{1}, y^{2}$ and $y^{3}$. Hence $p_{j}(y)=1$ and each player gets his demand $y_{j}$ with certainty, so that only deviations in which a player increases his demand has to be considered. Since $y_{i}<z_{i}$ for all $i$, we have that $y_{j}+y_{k}<v(j k)$ for any pair $j, k$. Suppose that player 3 increases his demand to $\widetilde{z}_{3}=v(13)-y_{1}$. Since $v(13)-y_{1} \leq v(23)-y_{2}$, then any twoplayer coalition can still fulfill the demands, so that player 3 receives this demand with probability $\frac{2}{3}$. Hence $y_{3}$ is at least as good as $\widetilde{z}_{3}$ if

$$
\frac{2}{3} \widetilde{z}_{3}=\frac{2}{3}\left[v(13)-y_{1}\right] \leq y_{3}
$$

Since $M_{3}<z_{3}$ and thus $y_{3}=\left(\lambda_{1}+\lambda_{2}\right) z_{3}+\lambda_{3} M_{3}>\lambda_{1} z_{3}+\left(1-\lambda_{1}\right) M_{3}$, by applying $y_{1}=\lambda_{1} M_{1}+\left(\lambda_{2}+\lambda_{3}\right) z_{1}=\lambda M_{1}+\left(1-\lambda_{1}\right) z_{1}$, it follows that this holds (with strict inequality) if

$$
2\left[v(13)-\lambda_{1} M_{1}-\left(1-\lambda_{1}\right) z_{1}\right] \leq 3\left[\lambda_{1} z_{3}+\left(1-\lambda_{1}\right) M_{3}\right]
$$

Define, for $\lambda_{1} \in[0,1]$,

$$
\begin{aligned}
& f\left(\lambda_{1}\right)=2\left[v(13)-\lambda_{1} M_{1}-\left(1-\lambda_{1}\right) z_{1}\right], \\
& g\left(\lambda_{1}\right)=3\left[\lambda_{1} z_{3}+\left(1-\lambda_{1}\right) M_{3}\right] .
\end{aligned}
$$

Then $f(0)=2\left[v(13)-z_{1}\right]=2 z_{3}<3 M_{3}=g(0)$. Further, $f(1) \leq g(1)$ if

$$
\begin{aligned}
\left.2\left[v(13)-M_{1}\right)\right] & =2[v(13)-v(N)+v(23)] \\
& \leq 3 z_{3}=3 \frac{v(13)+v(23)-v(12)}{2} .
\end{aligned}
$$

This reduces to $v(13)+v(23)+3 v(12) \leq 4 v(N)$, which is true with strict inequality because $v(13)+v(23)+2 v(12)<3 v(N)$ and $v(12)<v(N)$. Because of the linearity of the functions $f$ and $g$ in $\lambda_{1}$, it follows that $f\left(\lambda_{1}\right)<g\left(\lambda_{1}\right)$ for all $0 \leq \lambda_{1} \leq 1$, showing that $y_{3}$ is strictly better than $\widetilde{z}_{3}$. 
Next, if player 3 increases his demand to $\widehat{z}_{3}=v(23)-y_{2} \geq v(13)-y_{1}$, then coalition $\{1,3\}$ can not form when $v(23)-y_{2}>v(13)-y_{1}$, and player 3 gets $\widehat{z}_{3}$ with probability $\frac{1}{2}$. We have to show that $\frac{1}{2} \widehat{z}_{3} \leq y_{3}$. Since $y_{3}>\lambda_{2} z_{3}+\left(1-\lambda_{2}\right) M_{3}$ and $y_{2}=\lambda_{2} M_{2}+\left(1-\lambda_{2}\right) z_{2}$, this holds (with strict inequality) if

$$
v(23)-\lambda_{2} M_{2}-\left(1-\lambda_{2}\right) z_{2} \leq 2\left[\lambda_{2} z_{3}+\left(1-\lambda_{2}\right) M_{3}\right] .
$$

Now, define for $\lambda_{2} \in[0,1]$,

$$
\begin{aligned}
& f\left(\lambda_{2}\right)=v(23)-\lambda_{2} M_{2}-\left(1-\lambda_{2}\right) z_{2}, \\
& g\left(\lambda_{2}\right)=2\left[\lambda_{2} z_{3}+\left(1-\lambda_{2}\right) M_{3}\right] .
\end{aligned}
$$

Then $f(0)=v(23)-z_{2}=z_{3}<2 M_{3}=g(0)$. Further, $f(1) \leq g(1)$ if

$$
v(23)-M_{2}=v(23)-v(N)+v(13) \leq 2 z_{3}=v(13)+v(23)-v(12),
$$

which is true with strict inequality because $v(12)<v(N)$. Since the functions $f$ and $g$ are linear in $\lambda_{2}$, it follows that $f\left(\lambda_{2}\right)<g\left(\lambda_{2}\right)$ for all $0 \leq \lambda_{2} \leq 1$, showing that $y_{3}$ is strictly better than $\widehat{z}_{3}$. Hence $y_{3}$ is the unique element of $\beta_{3}(y)$. Analogously it follows that $y_{j}$ is the unique element of $\beta_{j}(y)$ for $j=1,2$, showing that $y$ is a strict stable demand vector. This proves case (iii) and the proposition.

Observe that the proof shows that any vector $x \in W(N, v)$ is a strict stable demand vector in case $|K(N, v)|=3$, irrespective whether or not the condition $v(i j) \leq v(N)$ for each pair $i, j$ holds with strict inequality. When $|K(N, v)|=1$, say $K(N, v)=\{i\}$, then $y^{i}$ is strict stable when $v(i j)<v(N)$ for $j \neq i$. Finally, in case $|K(N, v)|=2$, say $K(N, v)=\{i, j\}$, then $x \in W(N, v)$ is strict stable when $x$ is not an extreme point or the average $\frac{1}{2}\left(y^{i}+y^{j}\right)$ of the two extreme points. When $x$ is one of these three points, then $x$ strict stableness also requires that $v(i j)<v(N)$.

The results of Propositions 1, 3 and 4 can be summarized as follows.

Theorem 2. Let $(N, v)$ be a superadditive three-player game. If the core is not empty, then $C(N, v) \subseteq B(N, v)$. If the core is empty and

$$
v(12)+v(13)+v(23)+v(j k) \geq 3 v(N), \text { for each pair }\{j, k\},
$$

then the vNM vector $z$ is a stable demand vector. Finally, if the core is empty and this condition does not hold, then $W(N, v) \subseteq B(N, v)$.

We conclude this discussion on superadditive three-player games with Example 3 from Agastya [1]. Its purpose is twofold. First it shows that some stable demands may not be aspirations. Secondly, it demonstrates how relaxing the assumption of the smallest money unit results in the existence of (a) strict stable demand vector(s). 
Example 3. Let $N=\{1,2,3\}$ and let the characteristic function of the game be given by

$$
v(S)= \begin{cases}4, & \text { if } S=N \\ 3, & \text { if }|S|=2, \\ 0, & \text { otherwise }\end{cases}
$$

If the smallest money unit is $\delta=1$, i.e. $X_{i}=\{1,2,3,4\}$ for all $i \in N$, then, as is shown in [1], the strict stable demand set is empty. Suppose now that there is no smallest money unit. The vNM vector $z$ is given by $\left(\frac{3}{2}, \frac{3}{2}, \frac{3}{2}\right)$. By Proposition 3, $z$ is stable, but not strict. It can be checked that the vector $x^{*}=\left(\frac{4}{3}, \frac{4}{3}, \frac{4}{3}\right)$ is strict stable. Observe that $x^{*}$ is not an aspiration, since $x^{*}(S)<v(S)$ for all $S$, such that $|S|=2$.

5.2. Three-player/three-cake problems. For the problems of this type the characteristic function is given by $v(S)>0$ if and only if $|S|=$ 2 , and zero otherwise (and thus also $v(N)=0$ ). Thus the conditions of Proposition 3 are satisfied, implying that the $\mathrm{vNM}$ vector $z$ is a strict stable demand vector, provided $z_{i}, z_{j}, z_{k}>0$. In the following we consider the question of what other vectors are strict stable. Clearly, demand vectors $x$ of the form $x_{i}<z_{i}$ for all $i$ or $x_{i}>z_{i}$ for all $i$ can not be strictly stable. Neither can the ones of the type $\left(x_{i} \neq z_{i}, x_{j}=z_{j}\right.$, $\left.x_{k}=z_{k}\right)$. A little reflection shows that the ones of the type $\left(x_{i} \neq z_{i}\right.$, $\left.x_{j} \neq z_{j}, x_{k}=z_{k}\right)$ are not strict stable either. Thus, whenever one of the players, say player $i$, credibly demands $z_{i}$, he enforces players $j$ and $k$ to demand their $z_{j}$ and $z_{k}$. Hence, the solution $\left(z_{i}, z_{j}, z_{k}\right)$ seems particularly attractive.

The only two remaining possibilities for stable demand vectors are $\left(x_{i}>z_{i}, x_{j}>z_{j}, x_{k}<z_{k}\right)$ and $\left(x_{i}<z_{i}, x_{j}<z_{j}, x_{k}>z_{k}\right)$. In the first case, we have that

$$
x_{i}+x_{j}>z_{i}+z_{j}=v(i j),
$$

while stableness requires that

$$
\left\{\begin{array}{l}
x_{i}+x_{k}=v(i k), \\
x_{j}+x_{k}=v(j k) .
\end{array}\right.
$$

Similarly, the second case results in

$$
\left\{\begin{array}{l}
x_{i}+x_{j}<v(i j), \\
x_{i}+x_{k}=v(i k), \\
x_{j}+x_{k}=v(j k) .
\end{array}\right.
$$

Consequently for both these cases stableness requires that

$$
v(i k)-x_{i}=v(j k)-x_{j}
$$


or equivalently (using the definition of $z_{i}$ and $z_{j}$ )

$$
z_{i}-x_{i}=z_{j}-x_{j}
$$

Thus both players $i$ and $j$ gain (lose) by the same amount as compared to $z_{i}$ and $z_{j}$. Consequently, player $k$ loses (gains) by the same amount. Let $\alpha=x_{i}-z_{i}=x_{j}-z_{j}=z_{k}-x_{k}$, and suppose that $i$ and $j$ are such that they gain (lose) simultaneously. Then we have the following result.

Theorem 3. Let $(N, v)$ be a three-player/three-cake problem such that $z_{i}, z_{j}, z_{k}>0$. Then all strict stable demand vectors have the form $\left(z_{i}+\alpha, z_{j}+\alpha, z_{k}-\alpha\right)$, where

$$
\alpha \in\left(-\frac{1}{7} \min \left\{z_{i}, z_{j}\right\}, 0\right] \cup\left(\frac{1}{7} \max \left\{z_{i}, z_{j}\right\}, z_{k}\right) .
$$

Proof. It has been shown already above that any strict stable demand vector must be of the form $\left(z_{i}+\alpha, z_{j}+\alpha, z_{k}-\alpha\right)$. It remains to show that such a vector is indeed a stable demand vector iff $\alpha \in$ $\left(-\frac{1}{7} \min \left\{z_{i}, z_{j}\right\}, 0\right] \cup\left(\frac{1}{7} \max \left\{z_{i}, z_{j}\right\}, z_{k}\right)$.

First observe that regardless of whether $\alpha$ is positive or negative, player $k$ has no profitable deviations from $x^{*}$. Thus we have to check only for possible deviations of the players $i$ and $j$. If $\alpha>0$, then $p_{i}(x)=p_{j}(x)=\frac{1}{2}$. Consider player $i$. Clearly, the only profitable adjustment for player $i$ involves a decrease of his demand $x_{i}$ to $y_{i}=$ $v(i j)-x_{j}$, which he then will receive with probability $\frac{2}{3}$. Since $v(i j)-$ $x_{j}=v(i j)-\left[v(j k)-x_{k}\right]=v(i j)-v(j k)+z_{k}-\alpha=z_{i}-\alpha, x_{i}$ is the unique best reply of player $i$ to $x$ if and only if

$$
\frac{1}{2}\left(z_{i}+\alpha\right)>\frac{2}{3}\left(z_{i}-\alpha\right)
$$

which yields $\alpha>\frac{1}{7} z_{i}$. Analogously we have the restriction $\alpha>\frac{1}{7} z_{j}$ for player $j$, which gives the lower bound $\alpha>\frac{1}{7} \max \left\{z_{i}, z_{j}\right\}$. On the other hand we should have that $\alpha<z_{k}$, since $x_{k}=0$ when $\alpha=z_{k}$ and then any demand of player $k$ is a best reply to $x$. In case $z_{k} \leq \frac{1}{7} \min \left\{z_{i}, z_{j}\right\}$, conclude that there are no strict stable demand vectors with $\alpha>0$.

If $\alpha<0$, then $p_{i}(x)=p_{j}(x)=\frac{2}{3}$. It is easily checked that the only possible profitable adjustment of player $i$ involves an increase in his demand from $x_{i}$ to $y_{i}=v(i j)-x_{j}=v(i j)-\left[v(j k)-x_{k}\right]=v(i j)-$ $v(j k)+z_{k}-\alpha=z_{i}-\alpha$, which he then will receive with probability $\frac{1}{2}$. Hence $x_{i}$ is the unique best reply of player $i$ to $x$ if and only if

$$
\frac{2}{3}\left(z_{i}+\alpha\right)>\frac{1}{2}\left(z_{i}-\alpha\right),
$$


or equivalently $\alpha>-\frac{1}{7} z_{i}$. Analogously we have the restriction $\alpha>$ $-\frac{1}{7} z_{j}$ for player $j$, which gives the lower bound $\alpha>-\frac{1}{7} \min \left\{z_{i}, z_{j}\right\}$ on $\alpha<0$. Finally, note that the upper bound on $\alpha$ in this case equals zero, since $z$ is strict stable by Proposition 3 .

Observe that all strict stable demands with $\alpha>0$ are aspirations, whereas those with $\alpha<0$ are not, since $x_{i}+x_{j}<v(i j)$. Moreover no strict stable demand vector is an imputation (even not in its expected form), since $v(N)=0 .{ }^{6}$

\section{Further Examples}

6.1. Market games. Consider the following model of a market. The set of players $N$ is partitioned in two classes: sellers $L$ and buyers $M$. Each seller $i \in L$ has one unit of an indivisible good, to which he attaches a reservation price $w_{i}^{L}$. Every buyer $j \in M$ wants to buy exactly one unit of the good and has a reservation price of $w_{j}^{M}$. Suppose that all players submit their buying or selling prices $x_{k}$, where $k \in N$, to the central authority, who then matches the players according to some minimal partition. From Proposition 1 it follows that any core allocation is stable. On the other hand, it is well known that any competitive equilibrium is in the core of the corresponding transferable utility game. Therefore, all competitive equilibria of a market satisfy the stability requirements imposed by $B(N, v)$. The converse of Proposition 1 is not always true, as examples below will show. That is, there can be many allocations that are, in fact, stable demands, but do not belong to the core of the corresponding market game.

In this section we show that many interesting conventions in the above market game can be captured with the help of the framework developed in Section 2. Let us start with the simplest case, i.e. a one seller/two-buyer model. Specifically, let $L=\{1\}$ and $M=\{2,3\}$, that is player 1 is a seller and players 2 and 3 are buyers. Suppose that $w_{1}=0$ and $w_{2}, w_{3}>0$ (for simplicity we suppress the superscripts $L$ and $M)$. We will consider two instances: when buyers' valuations are identical and when they are not.

\footnotetext{
${ }^{6}$ There seems to be no consensus as how to define $v(N)$ in three-player/threecake problems. One can assume (as we did) $v(N)=0$; the other possibility would be to define $v(N)=\max _{|S|=2} v(S)$, see van der Laan and Houba [13]. In that case the stable solutions derived in Theorem 3 in their expected form can possibly be imputations. However, the theorem itself may be invalidated, since one also has to account for the possibility that the grand coalition forms and all demands are satisfied with probability one.
} 
Example 4. Let $w_{2}=w_{3}=w$. The characteristic function of the game becomes

$$
v(S)= \begin{cases}w, & \text { if } S \in\{\{12\},\{13\}, N\} \\ 0, & \text { otherwise }\end{cases}
$$

It is easy to see that no demand vector $x$, such that $x_{2} \neq x_{3}$ can be strict stable. Moreover, no $x>>0$, such that $x(N)=w$ is strict stable either, since player 1 has a profitable increase in demand that is satisfied with probability one. Thus, the grand coalition is never 'strict stable' and the game is essentially a three-player/three-cake problem. Analogously to Theorem 3, it follows that all strict stable demand vectors have the form $x^{*}=(w-\alpha, \alpha, \alpha)$, where $\alpha \in(0, w)$. For any $x^{*}$ it holds that $\Pi\left(x^{*}\right)=\{\{12,3\},\{13,2\}\}$ and $p_{1}\left(x^{*}\right)=1, p_{2}\left(x^{*}\right)=p_{3}\left(x^{*}\right)=\frac{1}{2}$.

In contrast, the core, kernel and nucleolus of the game consist of the single imputation $x=(w, 0,0)$. The latter is not a strict stable demand vector and, hence, cannot arise as a convention in the sense of Young [16]. The Shapley value of the game, which is given by $\left(\frac{2 w}{3}, \frac{w}{6}, \frac{w}{6}\right)$, is not stable either. It can be checked that every (strict) stable demand vector is an aspiration. However, for no $\alpha>0$ does a stable demand $x^{*}$ belong to the aspiration bargaining set, or the bargaining set of Aumann and Maschler, or the vNM stable set. The reason being that all these solution concepts ignore the nature of random matching among players. By applying Proposition 2, it can be seen that all (strict) stable solutions are efficient in their expected form. Graphically the set of all expected payoff vectors corresponding to strict stable demands (also including demands $(w, 0,0)$ and $(0, w, w))$ is shown by a solid line in the figure below.

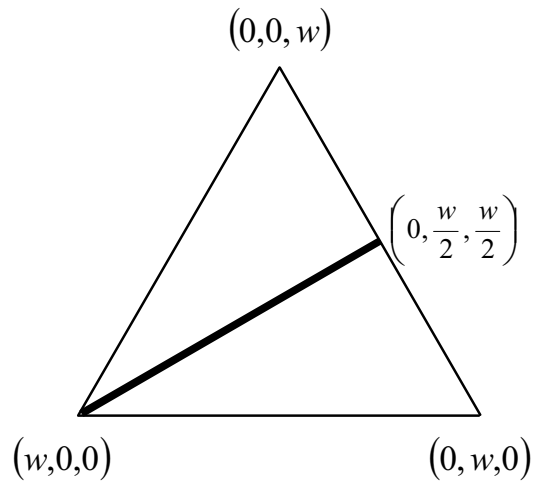

Figure 2: The set of strict stable demand vectors in expected form when $w_{2}=w_{3}=w$.

Interestingly in its expected form, each vector $x^{*}$ is a member of a vNM stable set, but not of the (aspiration) bargaining set (the latter 
is singleton point $\{(w, 0,0)\})$. For $\alpha=\frac{w}{3}$ we have that the vector of expected payoffs corresponds to the Shapley value of the game.

Note how the standard theory of general equilibrium approaches the same problem. It is shown that both buyers will compete for the seller and hence undercut each other. Correspondingly, the seller will obtain the whole surplus from trade. The above example shows how the impossibility of communication and 'discussing' prices may lead to an outcome, in which not all surplus goes to the seller. This situation can easily be generalized for the case of $n$ identical buyers. Specifically, let $L=\{1\}, M=\{2, \ldots, n\}$ and assume, as before, $w_{1}=0$, $w_{2}=\ldots=w_{n}=w$. Then any strict stable demand has the form $x=(w-\alpha, \alpha, \ldots, \alpha)$, where $\alpha \in(0, w)$.

Our next example shows that if buyers' valuations are different strict stable demand vectors can be inefficient in two distinct ways: (i) with positive probability the buyer with the highest valuation does not obtain the good, and (ii) players' expected utilities do not sum to the worth of the grand coalition.

Example 5. Let $w_{2}<w_{3}$. The characteristic function of the game becomes

$$
v(S)= \begin{cases}w_{2}, & \text { if } S=\{12\} \\ w_{3}, & \text { if } S \in\{\{13\}, N\} \\ 0, & \text { otherwise. }\end{cases}
$$

Since in this case $z_{2}<0$, one cannot apply Theorem 3, as in the previous example. However, it is clear that at any strict stable demand $x$, each buyer enters the coalition with the seller. Moreover, players' demands must exhaust all gains from coalition formation. A little reflection shows that there is only one type of demand vectors that enjoy these conditions. They have the form $x^{*}=\left(w_{2}-\alpha, \alpha, w_{3}-w_{2}+\alpha,\right)$, where $\alpha \in\left(0, w_{2}\right)$. For any $x^{*}$, it holds that $\Pi\left(x^{*}\right)=\{\{12,3\},\{13,2\}\}$ and $p_{1}\left(x^{*}\right)=1, p_{2}\left(x^{*}\right)=p_{3}\left(x^{*}\right)=\frac{1}{2}$.

Observe that no allocation in the core of the above game is strict stable, since in any such allocation player 2 obtains zero. Moreover, for any $\alpha>0$ a stable demand vector $x^{*}$ is not an imputation any longer, even not in its expected form. Correspondingly, it is not a member of either core, or stable, or bargaining set and does not equal the Shapley value of the game. Nevertheless, all vectors $x^{*}$ can be interpreted as conventions, in which the coalition of buyers gains 'market power' and thus imposes losses on the single seller ${ }^{7}$. In its extreme form (when

\footnotetext{
${ }^{7}$ Typically, a related question - whether the seller can benefit from the presence of a 'weak' buyer (player 2 in our case) - is considered in the literature. There are models that answer it affirmatively, see Montero [14].
} 
$\alpha=w_{2}$ ) coalition $\{23\}$ keeps the price at zero. In this way, each of the buyers obtains the highest possible surplus with probability $\frac{1}{2}$, and no-one of them can profitably deviate from this convention if the seller approaches both buyers with equal probability. The generalization to the case of $n-1$ buyers with different valuations is also straightforward. Specifically, if $w_{2}<\ldots<w_{n}$ it can be shown that for any strict stable demand vector $x^{*}$ we have $x_{1}^{*}=w_{2}-\alpha, x_{2}^{*}=\alpha$ and for $j \in\{3, \ldots, n\}$ $x_{j}^{*}=w_{j}-w_{2}+\alpha$, where $\alpha \in\left(0, w_{2}\right)$. As a consequence, $x_{i}^{*}<x_{j}^{*}$ for any $i$ and $j$ such that $2 \leq i<j \leq n$.

Let us revert to the case of two buyers with different valuations. Before we stressed that no communication among players was possible. Suppose we relax this assumption and allow that players can make any kind of proposals to each other. Suppose further that player 1 wants to allocate his object by means of an English auction, in which all indeterminacies are resolved with the help of a lottery ${ }^{8}$. Then the 'fair' price in this auction is $w_{2}$. Correspondingly, player 1 would earn the surplus of $w_{2}$, player 3 would get $w_{3}-w_{2}$ and player 2 would obtain zero. However, if $w_{2} \geq \frac{w_{3}}{2}$ player 3 has incentives to propose to player 2 to keep the price at zero level. This would result in a lottery, in which both buyers obtain the object with probability $\frac{1}{2}$. Player 2 does not have any incentives not to agree to this proposal, since for any price $p \leq w_{2}$ player 3 in the English auction overbids player 2 . By agreeing to cooperate with player 3, player 2, however, obtains a strictly positive surplus in expected terms. This situation exemplifies the case, in which collusion between bidders in the English auction is particularly attractive. It seems that player 1, if he is 'rational', should never use the English auction in this case at all!

By introducing the possibility of making binding agreements, one can push the above logic even further. Observe that in the core, player 2 obtains zero surplus, and players 1 and 3 can divide the surplus $w_{3}-w_{2}$ in any way they like. However, if player 1 offers to player 3 less than $\frac{w_{3}}{2}$, player 3 can threaten to make a binding agreement with player 2 to keep the price at zero (or at $\epsilon$, which is sufficiently small). This threat is credible, because player 2 will gladly sign any binding agreement with player 3 . In fact, if $w_{2}>\frac{w_{3}}{2}$ then the core is not stable in the above sense at all, since player 3 always wants to collude with player 2 !

We conclude this part on market games with some remarks on the symmetry of (strict) stable solutions. From Example 4 it may seem that symmetric players must obtain the same payoff. However, this

\footnotetext{
${ }^{8}$ The results will not hold for a sealed bid auction, though.
} 
does not hold generally. It may well be the case that some (strict) stable demands involve asymmetric claims and payoffs of symmetric players. Consider, for instance, the market game where $L=\{1,2\}, M=\{3,4\}$, $w_{i}^{L}=0$ for all $i \in L$ and $w_{j}^{M}=1$ for all $j \in M$. Under these conditions, clearly all sellers are symmetric and all buyers are symmetric. However, it can be verified that the demand vector $x=\left(\frac{3}{4}, \frac{2}{3}, \frac{1}{4}, \frac{1}{3}\right)$ is strict stable $^{9}$. Moreover, $x$ is not in the core of the game, which is given by the set $\left\{(\alpha, \alpha, 1-\alpha, 1-\alpha) \in \mathbb{R}^{4} \mid \alpha \in[0,1]\right\}$.

6.2. Apex games. The characteristic function of the apex game of player $j$ and coalition $J \subset N \backslash\{j\}$ with $J \neq \varnothing$ is given by

$$
v(S)= \begin{cases}1, & \text { if either } J \subseteq S \text { or both } S \ni j \text { and } S \cap J \neq \varnothing, \\ 0, & \text { otherwise. }\end{cases}
$$

Thus, the worth $v(N)$ can be earned by player $j$ together with at least one player $i \in N \backslash\{j\}$ or by all players in $J$ cooperating together. Clearly apex games are superadditive and all players in $J$ are symmetric. In what follows, without loss of generality we will consider the apex game of player 1 only; thus $J=N \backslash\{1\}$. To avoid degeneracies, we also assume that $|N| \geq 3$.

We will show now that strict stable demand vectors in apex games can be characterized in a neat way. Let $x$ be a strict stable demand and consider the following three possibilities:

i) $\sum_{i \in J} x_{i}>1$.

ii) $\sum_{i \in J} x_{i}<1$.

iii) $\sum_{i \in J} x_{i}=1$.

First we consider case (i). Since coalition $J$ is not feasible, strictness implies that $x_{i}>0$ for all $i$ and that every player $i \in J$ can form a coalition with the apex player, otherwise its probability is equal to zero. Moreover, for $i \in J$ we should have that $x_{1}+x_{i}=1$, since $i$ can increase its demand if $x_{1}+x_{i}<1$. Consequently, all strict stable demands have the form $(1-\alpha, \alpha, \ldots, \alpha)$ for some $\alpha<1$. To find the lower bound on $\alpha$, note that if $\alpha \geq \frac{1}{n-2}$, then clearly no player $i \in J$ can benefit from changing his demand. If, however, $\alpha<\frac{1}{n-2}$, we need to ensure that no $i \in J$ has a profitable reduction in demand to $y_{i}=1-\alpha(n-2)$. That is, if

$$
\frac{2(1-\alpha(n-2))}{n}<\frac{\alpha}{n-1}
$$

\footnotetext{
${ }^{9}$ Since $p_{i}(x)=1$ for all $i \in N$ one only has to check whether players 2 and 3 do not want to increase their demands to $\frac{3}{4}$ and $\frac{1}{3}$, respectively.
} 
or

$$
\frac{2(n-1)}{2 n^{2}-5 n+4}<\alpha
$$

holds. This condition gives the lower bound on $\alpha$, which decreases with $n$.

If $x$ is a strict stable demand vector in case (ii), it should also have the form $(1-\alpha, \alpha, \ldots, \alpha)$. To see why, note that if there is $i \in J$ such that $x_{1}+x_{i}<1$, then player $i$ can increase his demand up to $y_{i}=\min \left\{1-x_{1}, 1-\sum_{j \in J \backslash\{i\}} x_{j}\right\}-\epsilon$, where $\epsilon$ is positive and small enough $^{10}$ and get it satisfied with probability $p_{i}(x)$. Thus, for all $i \in J$ we have $x_{1}+x_{i} \geq 1$. If, however, $x_{1}+x_{i}>1$ for some $i \in J$, then such player $i$ can increase his demand up to $y_{i}=\left(1-\sum_{j \in J \backslash\{i\}} x_{j}\right)-\epsilon$ and still get it with probability $p_{i}(x)$. Thus, for all $i \in J$ we have $x_{1}+x_{i} \leq 1$. Hence the equality $x_{1}+x_{i}=1$. Allowing now $x_{i}=\alpha$ for all $i \in J$ we need to deter possible deviations to $y_{i}=1-\alpha(n-2)$. This results in the following condition

$$
\frac{2 \alpha}{n}>\frac{1-\alpha(n-2)}{n-1}
$$

After simplification we obtain the lower bound $\alpha>\frac{n}{n^{2}-2}$. The upper bound $\alpha<\frac{1}{n-1}$ follows from the condition $\sum_{i \in J} x_{i}<1$.

Case (iii) requires that there exists at least one player $i \in J$ such that $x_{1}+x_{i}=1$. Clearly, $i$ solves

$$
i \in \arg \min _{j \in J}\left\{x_{2}, \ldots, x_{n}\right\} \text {. }
$$

Without loss of generality, let $i=2$ and $x_{2} \leq \ldots \leq x_{n}$. Clearly, player 2 does not have profitable deviations. In order to deter possible deviations of other players in $J \backslash\{2\}$ we need to have $x_{j}>\frac{4}{3} x_{2}$ for all $j \in J$ such that $x_{j}>x_{2}$. As for player 1 , we need

$$
\frac{x_{1}}{2}>\left(1-x_{k}\right) \frac{k-1}{k}
$$

for all $k=3, \ldots, n$. In such a case the symmetry among the players in $J$ need not hold anymore. For instance, let $n=4$ and consider the vector of demands $x^{*}=\left(\frac{27}{30}, \frac{3}{30}, \frac{13}{30}, \frac{14}{30}\right)$. It can be verified that $x^{*}$ is strict stable, yet all symmetric players $(2,3$ and 4$)$ submit different demands and obtain different expected payoffs.

By applying Proposition 2, we can see that the efficiency of strict stable demands in their expected forms is achieved only in cases (i)

\footnotetext{
${ }^{10}$ This ensures that $\sum_{j \in J \backslash\{i\}} x_{j}+x_{i}<1$ holds.
} 
and (iii). The following symmetric solution $x^{*}$ that belongs to the last case has already received some attention in the literature:

$$
x_{1}^{*}=\frac{n-2}{n-1} \text { and } x_{i}^{*}=\frac{1}{n-1} \text { for all } i \in J .
$$

As it was shown by Bennett and van Damme [5], this demand vector is a unique subgame perfect equilibrium in a certain proposal making model. Moreover, in its expected form $x_{i}^{*}$ corresponds to the Shapley value of player $i \in N$, see van den Brink [9]. Note that for $N=\{1,2,3\}$ the apex game becomes a superadditive three-player game and the demand vector $x^{*}$ equals the vNM vector $z=\left(\frac{1}{2}, \frac{1}{2}, \frac{1}{2}\right)$ in this case. Consequently, $x^{*}$ is also strict stable according to Proposition 3 .

Finally, we will illustrate that the set $\widetilde{B}(N, v)$ does not have to be convex. For the apex game with $n=4$ take $x^{*}=\left(\frac{5}{8}, \frac{3}{8}, \frac{3}{8}, \frac{3}{8}\right)$ and $y^{*}=\left(\frac{10}{11}, \frac{1}{11}, \frac{5}{11}, \frac{5}{11}\right)$. Both $x^{*}$ and $y^{*}$ are strict stable demands, it is easily verified that $x^{*}$ belongs to case (i) above and $y^{*}$ to case (iii). Consider a convex combination $w=\gamma x^{*}+(1-\gamma) y^{*}$ with $\gamma=\frac{3}{4}$. It can be checked that for $j \in\{3,4\}$ the condition $w_{j}>\frac{4}{3} w_{2}$ is violated and, hence, $w$ is not a stable demand vector.

\section{REFERENCES}

[1] Agastya, M. (1997) "Adaptive Play in Multiplayer Bargaining Situations", Review of Economic Studies, 64, 411-26.

[2] Aumann, R. J., M. Maschler "The Bargaining Set for Cooperative Games", in Advances in Game Theory, M. Dresher, L. S. Shapley and A. W. Tucker (eds.), Princeton Univ. Press 1964, 443-76.

[3] Bennett, E. (1983) "The Aspirations Approach to Predicting Coalition Formation and Payoff Distribution in Side Payment Games", International Journal of Game Theory, 12, 1-28.

[4] Bennett, E. (1997) "Multilateral Bargaining Problems", Games and Economic Behavior, 19, 151-79.

[5] Bennett, E., E. van Damme "Demand Commitment Bargaining: The Case of Apex Games", in Game Equilibrium Models, Vol 3, Strategic Bargaining, R. Selten (ed.), Springer-Verlag 1991, 118-40.

[6] Bennett, E., M. Maschler, W. Zame (1997) "A Demand Adjustment Process", International Journal of Game Theory, 26, 423-38.

[7] Bennett, E., W. Zame (1988) "Bargaining in Cooperative Games", International Journal of Game Theory, 17, 279-300.

[8] Binmore, K. "Bargaining in Cooperative Games", in Game Theoretic Models and Bargaining, A. Roth (ed.), Cambridge Univ. Press 1986, 269-304.

[9] Brink, van den R. (2002) "The Apex Power Measure for Directed Networks", Social Choice and Welfare, 19, 845-67.

[10] Cross, J. G. (1967) "Some Theoretical Characteristics of Economic and Political Coalitions", Journal of Conflict Resolution, 11, 184-95. 
[11] Davis, M., M. Maschler (1965) "The Kernel of a Cooperative Game", Naval Research Logistic Quarterly, 12, 223-59.

[12] Krishna, V., R. Serrano (1996) "Multilateral Bargaining", Review of Economic Studies, 63, 61-80.

[13] Laan, G. van der, H. Houba (2002) "One Seller/Two Buyer Markets with Buyer Externalities and (Im)perfect Competition", International Game Theory Review, 4, 141-64.

[14] Montero, M. (2000) Endogenous Coalition Formation and Bargaining, PhD Dissertation, Tilburg University.

[15] Rubinstein, A. (1982) "Perfect Equilibrium in a Bargaining Model", Econometrica, 50, 97-109.

[16] Young, P. (1993) "The Evolution of Conventions", Econometrica, 61, 57-84.

FEWEB, Vrije Universiteit Amsterdam, De Boelelaan 1105, $1081 \mathrm{HV}$ Amsterdam, The Netherands

E-mail address, G. van der Laan: glaan@feweb.vu.nl

E-mail address, V. Pruzhansky: pruzhansky@tinbergen.nl 